\title{
Existence of solutions of a second-order impulsive differential equation
}

\author{
Zhiguo Luo', Jingli Xie ${ }^{2^{*}}$ and Guoping Chen ${ }^{2}$
}

"Correspondence: xiejingli721124@163.com

${ }^{2}$ College of Mathematics and Statistics, Jishou University, Jishou, Hunan 416000, P.R. China Full list of author information is available at the end of the article

\begin{abstract}
This paper is concerned with the existence of solutions of a second-order impulsive differential equation with mixed boundary condition. We obtain sufficient conditions for the existence of a unique solution, at least one solution, at least two solutions and infinitely many solutions, respectively, by using critical point theorems. The main results are also demonstrated with examples.
\end{abstract}

MSC: 34B15; 34B18; 34B37; 58E30

Keywords: impulsive differential equation; critical point theory; existence of solutions

\section{Introduction}

Nowadays, with the rapid development of science and technology, many people have realized that the theory of impulsive differential equations is not only richer than the corresponding theory of differential equations but it also represents a more natural framework for mathematical modeling of real world phenomena. Hence, it has become an effective tool to study some problems of biology, medicine, physics and so on [1,2]. Significant progress has been made in the theory of systems of impulsive differential equations in recent twenty years (see [3-8] and the references cited therein). We generally consider impulses in the position $u$ and $u^{\prime}$ for the second-order differential equation $u^{\prime \prime}=f\left(t, u, u^{\prime}\right)$. However, it is well known that in the motion of spacecraft instantaneous impulses depend on the position, which results in jump discontinuities in velocity, with no change in the position. This motivates us to consider the following second-order impulsive differential equation:

$$
\left\{\begin{array}{l}
-u^{\prime \prime}(t)+g(t) u(t)=f(t, u(t)), \quad t \neq t_{j}, t \in J=[0, T], T>0, \\
-\Delta u^{\prime}\left(t_{j}\right)=I_{j}\left(u\left(t_{j}\right)\right), \quad j=1,2, \ldots, m, \\
u(0)=0, \quad \alpha u(T)+\beta u^{\prime}(T)=0,
\end{array}\right.
$$

where $f \in C(J \times R, R), g \in L^{\infty}[0, T], g(t)>0, I_{j} \in C(R, R), 0=t_{0}<t_{1}<t_{2}<\cdots<t_{m}<t_{m+1}=$ $T, \alpha, \beta$ are constants with $\alpha \geq 0, \beta>0$, and the operator $\Delta$ is defined as $\Delta u^{\prime}\left(t_{j}\right)=u^{\prime}\left(t_{j}^{+}\right)-$ $u^{\prime}\left(t_{j}^{-}\right)$, where $u^{\prime}\left(t_{j}^{+}\right)\left(u^{\prime}\left(t_{j}^{-}\right)\right)$denotes the right-hand (left-hand) limit of $u^{\prime}$ at $t_{j}$.

In recent years, some classical tools such as some fixed point theorems in cones, topological degree theory and the upper and lower solutions method combined with the monotone iterative technique [9-14] have been widely used to get solutions of impulsive differential equations. On the other hand, in the last few years, some researchers have studied

@2014 Luo et al.; licensee Springer. This is an Open Access article distributed under the terms of the Creative Commons Attribution License (http://creativecommons.org/licenses/by/2.0), which permits unrestricted use, distribution, and reproduction in any medium, provided the original work is properly cited. 
the existence of solutions for impulsive differential equations with boundary conditions via variational methods [15-19]. In this paper, we consider (1.1) by using critical point theory and variational methods.

The rest of this paper is organized as follows. In Section 2 we present several important lemmas. In Section 3, we present existence results of equation (1.1) by using critical point theory and variational methods.

\section{Preliminaries}

In the following, we first introduce some notations and some necessary definitions.

Definition 2.1 [20] Let $X$ be a real reflexive Banach space. For any sequence $\left\{u_{k}\right\} \subset X$, if $\left\{\varphi\left(u_{k}\right)\right\}$ is bounded and $\varphi^{\prime}\left(u_{k}\right) \rightarrow 0$ as $k \rightarrow \infty$ possesses a convergent subsequence, then we say that $\varphi$ satisfies the Palais-Smale condition (PS condition).

Definition 2.2 [20] Let $\varphi: X \rightarrow R$ be differentiable and $c \in R$. We say that $\varphi$ satisfies the (PS) $)_{c}$ condition if the existence of a sequence $\left\{u_{k}\right\}$ in $X$, such that $\varphi\left(u_{k}\right) \rightarrow c, \varphi^{\prime}\left(u_{k}\right) \rightarrow 0$ as $k \rightarrow \infty$, implies that $c$ is a critical value of $\varphi$.

It is clear that the PS condition implies the $(\mathrm{PS})_{c}$ condition for each $c \in R$.

Lemma 2.3 [21] Let $H$ be a Hilbert space and $a: H \times H \rightarrow R$ be a bounded bilinear form. If $a$ is coercive, i.e., there exists $\alpha>0$ such that $a(u ; u) \geq \alpha\|u\|^{2}$ for every $u \in H$, then for any $\sigma \in H^{\prime}$ (the conjugate space of $H$ ), there exists a unique $u \in H$ such that

$$
a(u, v)=(\sigma, v) \text { for every } v \in H .
$$

Moreover, if $a$ is also symmetric, then the functional $\varphi: H \rightarrow R$ defined by $\varphi=\frac{1}{2} a(v, v)-$ $(\sigma, v)$ attains its minimum at $u$.

Lemma 2.4 [20] If $\varphi$ is weakly lower semi-continuous on a reflexive Banach space $X$ and has a bounded minimizing sequence, then $\varphi$ has a minimum on $X$. The existence of a bounded minimizing sequence will be in particular insured when $\varphi$ is coercive, i.e., such that $\varphi(u) \rightarrow+\infty$ if $\|u\| \rightarrow \infty$.

Lemma 2.5 [22] For the functional $F: M \subseteq X \rightarrow R$ with $M$ not empty, $\min _{u \in M} F(u)=a$ has a solution in case the following hold:

(i) $X$ is a real reflexive Banach space;

(ii) $M$ is bounded and weakly sequentially closed;

(iii) $F$ is weakly sequentially lower semi-continuous on $M$, i.e., by definition, for each sequence $\left\{u_{k}\right\}$ in $M$ such that $u_{k} \rightarrow u$ as $k \rightarrow \infty$, we have $F(u) \leq \underline{\lim }_{k \rightarrow \infty} F\left(u_{k}\right)$.

Lemma 2.6 [20] Let $X$ be a Banach space and $\varphi \in C^{1}(X, R)$. Assume that there exist $u_{0} \in$ $X, u_{1} \in X$ and a bounded open neighborhood $\Omega$ of $u_{0}$ such that $u_{1} \in X \backslash \Omega$ and

$$
\inf _{\partial \Omega} \varphi>\max \left\{\varphi\left(u_{0}\right), \varphi\left(u_{1}\right)\right\}
$$

Let

$$
\Gamma=\left\{h \in C([0,1], X): h(0)=u_{0}, h(1)=u_{1}\right\}
$$


and

$$
c=\inf _{h \in \Gamma} \max _{s \in[0,1]} \varphi(h(s)) .
$$

If $\varphi$ satisfies the $(\mathrm{PS})_{c}$, then $c$ is a critical value of $\varphi$ and $c>\max \left\{\varphi\left(u_{0}\right), \varphi\left(u_{1}\right)\right\}$.

Lemma 2.7 [23] Let $X$ be an infinite dimensional Banach space and let $\varphi \in C^{1}(X, R)$ be even, satisfying the (PS), and $\varphi(0)=0$. If $X=V \oplus W$, where $V$ is finite dimensional, and $\varphi$ satisfies the following conditions:

(i) There exist constants $\rho, \sigma>0$ such that $\left.\varphi\right|_{\partial B_{\rho} \cap W} \geq \sigma$;

(ii) For each finite dimensional subspace $V_{1} \subset X$, there is an $R=R\left(V_{1}\right)$ such that $\varphi(u) \leq 0$ for every $u \in V_{1}$ with $\|u\|>R$;

then $\varphi$ possesses an unbounded sequence of critical values.

Let

$$
H^{1}([0, T])=\left\{u \in L^{2}([0, T]): u^{\prime} \in L^{2}([0, T])\right\}
$$

and

$$
H^{2}([0, T])=\left\{u \in L^{2}([0, T]): u^{\prime}, u^{\prime \prime} \in L^{2}([0, T])\right\} .
$$

Take $H=\left\{u \in H^{1}([0, T]): u(0)=0\right\}$. Then $H$ is a Hilbert space, and the inner product

$$
(u, v)=\int_{0}^{T} u^{\prime}(t) v^{\prime}(t) d t+\int_{0}^{T} g(t) u(t) v(t) d t
$$

induces the norm

$$
\|u\|=\left(\int_{0}^{T}\left(u^{\prime}(t)\right)^{2} d t+\int_{0}^{T} g(t) u^{2}(t) d t\right)^{\frac{1}{2}}
$$

For $u \in H^{2}([0, T])$, we have that $u$ and $u^{\prime}$ are both absolutely continuous and $u^{\prime \prime} \in$ $L^{2}([0, T])$, hence $\Delta u^{\prime}\left(t_{j}\right)=u^{\prime}\left(t_{j}^{+}\right)-u^{\prime}\left(t_{j}^{-}\right)=0$ for any $t \in J$. If $u \in H$, then $u$ is absolutely continuous and $u^{\prime} \in L^{2}([0, T])$. In this case, the one-side derivatives $u^{\prime}\left(t_{j}^{+}\right)$and $u^{\prime}\left(t_{j}^{-}\right)$may not exist. So, by a classical solution of (1.1), we mean a function $u \in C([0, T])$ satisfying the following conditions: For every $j=0,1, \ldots, m, u_{j}=\left.u\right|_{\left(t_{j}, t_{j+1}\right)} \in H^{2}\left(t_{j}, t_{j+1}\right) ; u$ satisfies the boundary condition of (1.1) and the first equation of $(1.1) ; u^{\prime}\left(t_{j}^{+}\right)$and $u^{\prime}\left(t_{j}^{-}\right), j=0,1, \ldots, m$, exist and the impulsive conditions of (1.1) hold.

Taking $v \in H$ and multiplying (1.1) by $v$ and integrating from 0 to $T$, we have

$$
-\int_{0}^{T} u^{\prime \prime}(t) v(t) d t+\int_{0}^{T} g(t) u(t) v(t) d t=\int_{0}^{T} f(t, u(t)) v(t) d t .
$$

This leads to

$$
\begin{aligned}
& \int_{0}^{T} u^{\prime}(t) v^{\prime}(t) d t+\int_{0}^{T} g(t) u(t) v(t) d t-\sum_{j=1}^{m} I_{j}\left(u\left(t_{j}\right)\right) v\left(t_{j}\right)+\frac{\alpha}{\beta} u(T) v(T) \\
& \quad=\int_{0}^{T} f(t, u(t)) v(t) d t
\end{aligned}
$$


Thus, a weak solution of (1.1) is a function $u \in H$ such that (2.1) holds for any $v \in H$. By the regularity theory, the weak solution is a classical solution. Now, we define $\varphi: H \rightarrow R$ by

$$
\begin{aligned}
\varphi(u)= & \frac{1}{2} \int_{0}^{T}\left[\left(u^{\prime}(t)\right)^{2}+g(t) u^{2}(t)\right] d t-\int_{0}^{T} F(t, u(t)) d t-\sum_{j=1}^{m} \int_{0}^{u\left(t_{j}\right)} I_{j}(t) d t \\
& +\frac{\alpha}{2 \beta} u^{2}(T),
\end{aligned}
$$

where $F(t, u(t))=\int_{0}^{u} f(t, s) d s$. Clearly, $\varphi$ is Fréchet differentiable at any $u \in H$ and

$$
\begin{aligned}
\varphi^{\prime}(u)(v)= & \lim _{h \rightarrow 0} \frac{\varphi(u+h v)-\varphi(u)}{h} \\
= & \int_{0}^{T}\left[u^{\prime}(t) v^{\prime}(t)+g(t) u(t) v(t)\right] d t-\int_{0}^{T} f(t, u(t)) v(t) d t \\
& -\sum_{j=1}^{m} I_{j}\left(u\left(t_{j}\right)\right) v\left(t_{j}\right)+\frac{\alpha}{\beta} u(T) v(T)
\end{aligned}
$$

for any $v \in H$. Obviously, $\varphi^{\prime}$ is continuous, and a critical point of $\varphi$ gives a weak solution of (1.1).

Lemma 2.8 If the function $u \in H$ is a critical point of the functional $\varphi$, then $u$ is a solution of system (1.1).

Proof Suppose that $u \in H$ is a critical point of the functional $\varphi$. Then, for any $v \in H$, one has

$$
\varphi^{\prime}(u)(v)=0 .
$$

From (2.3), one has

$$
\begin{aligned}
\int_{0}^{T} & {\left[u^{\prime}(t) v^{\prime}(t)+g(t) u(t) v(t)\right] d t-\int_{0}^{T} f(t, u(t)) v(t) d t-\sum_{j=1}^{m} I_{j}\left(u\left(t_{j}\right)\right) v\left(t_{j}\right)+\frac{\alpha}{\beta} u(T) v(T) } \\
= & -\sum_{j=1}^{m}\left(\Delta u^{\prime}\left(t_{j}\right)+I_{j}\left(u\left(t_{j}\right)\right)\right) v\left(t_{j}\right)+\int_{0}^{T}\left(-u^{\prime \prime}(t)+g(t) u(t)\right) v(t) d t \\
& -\int_{0}^{T} f(t, u(t)) v(t) d t+\left(u^{\prime}(T)+\frac{\alpha}{\beta} u(T)\right) v(T)
\end{aligned}
$$

Combining (2.4) and (2.5), one has

$$
\begin{aligned}
& -\sum_{j=1}^{m}\left(\Delta u^{\prime}\left(t_{j}\right)+I_{j}\left(u\left(t_{j}\right)\right)\right) v\left(t_{j}\right)+\int_{0}^{T}\left(-u^{\prime \prime}(t)+g(t) u(t)\right) v(t) d t \\
& -\int_{0}^{T} f(t, u(t)) v(t) d t+\left(u^{\prime}(T)+\frac{\alpha}{\beta} u(T)\right) v(T)=0, \quad \forall v \in H .
\end{aligned}
$$

For $j \in\{1,2, \ldots, m\}$, we choose $v \in H$ with $v(t)=0$ for every $t \in\left[0, t_{j}\right] \cup\left[t_{j+1}, T\right]$, then

$$
\int_{t_{j}}^{t_{j+1}}\left(-u^{\prime \prime}(t)+g(t) u(t)-f(t, u(t))\right) v(t) d t=0 .
$$


We get

$$
-u^{\prime \prime}(t)+g(t) u(t)=f(t, u(t)) \quad \text { a.e. } t \in\left(t_{j}, t_{j+1}\right) .
$$

Thus, $u$ satisfies the equation in (1.1).

Therefore, by (2.6) we have

$$
-\sum_{j=1}^{m}\left(\Delta u^{\prime}\left(t_{j}\right)+I_{j}\left(u\left(t_{j}\right)\right)\right) v\left(t_{j}\right)+\left(u^{\prime}(T)+\frac{\alpha}{\beta} u(T)\right) v(T)=0, \quad \forall v \in H .
$$

Next we prove that $u$ satisfies the impulsive and the boundary condition in (1.1). If the impulsive condition in (1.1) does not hold, then there exist some $j \in\{1,2, \ldots, m\}$ such that

$$
\Delta u^{\prime}\left(t_{j}\right)+I_{j}\left(u\left(t_{j}\right)\right) \neq 0 .
$$

Pick $v(t)=\prod_{i=0, i \neq j}^{m+1}\left(t-t_{i}\right)$, then

$$
\begin{aligned}
& -\sum_{j=1}^{m}\left(\Delta u^{\prime}\left(t_{j}\right)+I_{j}\left(u\left(t_{j}\right)\right)\right) v\left(t_{j}\right)+\left(u^{\prime}(T)+\frac{\alpha}{\beta} u(T)\right) v(T) \\
& =-\sum_{j=1}^{m}\left(\Delta u^{\prime}\left(t_{j}\right)+I_{j}\left(u\left(t_{j}\right)\right)\right) \prod_{i=0, i \neq j}^{m+1}\left(t_{j}-t_{i}\right)+\left(u^{\prime}(T)+\frac{\alpha}{\beta} u(T)\right) \prod_{i=0, i \neq j}^{m+1}\left(t_{m+1}-t_{i}\right) \\
& =-\left(\Delta u^{\prime}\left(t_{j}\right)+I_{j}\left(u\left(t_{j}\right)\right)\right) \prod_{i=0, i \neq j}^{m+1}\left(t_{j}-t_{i}\right) \neq 0 .
\end{aligned}
$$

This is a contradiction. So $u$ satisfies the impulsive condition in (1.1) and (2.7) implies

$$
\left(u^{\prime}(T)+\frac{\alpha}{\beta} u(T)\right) v(T)=0, \quad \forall v \in H .
$$

If $\alpha u(T)+\beta u^{\prime}(T) \neq 0$, then $\frac{\alpha}{\beta} u(T)+u^{\prime}(T) \neq 0$.

Pick $v(t)=\prod_{i=0}^{m}\left(t-t_{i}\right)$. One has

$$
\left(u^{\prime}(T)+\frac{\alpha}{\beta} u(T)\right) \prod_{i=0}^{m}\left(t_{m+1}-t_{i}\right) \neq 0 .
$$

This contradicts (2.10), so $u$ satisfies the boundary condition. Therefore, $u$ is a solution of system (1.1).

Lemma 2.9 If $u \in H$, then $\|u\|_{\infty} \leq T^{\frac{1}{2}}\|u\|$, where $\|u\|_{\infty}=\max _{t \in[0, T]}|u(t)|$.

Proof The proof follows easily from the Hölder inequality. The detailed argument is similar to the proof of Lemma 2.2 in [24], and we thus omit it here.

\section{Main results}

\subsection{Existence of a unique solution}

In this section we derive conditions under which system (1.1) admits a unique solution. 
Theorem 3.1 Assume that $d_{j}(j=1,2, \ldots, m)$ are fixed constants, $f(t, u)=\sigma(t) \in L^{2}(0, T)$ and $I_{j}(t)=d_{j}(j=1,2, \ldots, m)$, then system (1.1) has a unique solution $u$, and $u$ minimizes the functional (2.2).

Proof We define the bilinear form

$$
a: H \times H \rightarrow R, \quad a(u, v)=\int_{0}^{T}\left(u^{\prime}(t) v^{\prime}(t)+g(t) u(t) v(t)\right) d t+\frac{\alpha}{\beta} u(T) v(T)
$$

and the linear operator

$$
l: H \rightarrow R, \quad l(v)=\int_{0}^{T} \sigma(t) v(t) d t-\sum_{j=1}^{m} d_{j} v\left(t_{j}\right) .
$$

It is evident that $a$ is continuous and symmetric and $l$ is bounded. Moreover, $a$ is coercive. By Lemma 2.3, system (1.1) has a unique solution $u$, and $u$ minimizes the functional (2.2).

Example 3.1 Consider the following boundary value problem:

$$
\left\{\begin{array}{l}
-u^{\prime \prime}(t)+u(t)=0, \quad t \neq t_{1}, t \in J=[0,1] \\
-\Delta u^{\prime}\left(t_{1}\right)=1, \quad t_{1}=\frac{1}{2} \\
u(0)=0, \quad u(1)+u^{\prime}(1)=0
\end{array}\right.
$$

Here $g(t)=1, f(t, u)=0, I_{j}(u)=1, T=1, j=1, \alpha=1, \beta=1$. Applying Theorem 3.1, problem (3.1) has a unique solution. By simple calculations, we obtain $u(t)=\frac{1}{2} e^{-\frac{1}{2}}\left(e^{t}-e^{-t}\right), t \in\left[0, \frac{1}{2}\right]$, $u(t)=\frac{1}{2}\left(e^{\frac{1}{2}}-e^{-\frac{1}{2}}\right) e^{-t}, t \in\left[\frac{1}{2}, 1\right]$.

\subsection{Existence of at least one solution}

In this section we derive conditions under which system (1.1) admits at least one solution. For this purpose, we introduce the following assumption.

(H1) There exist $a, b, a_{j}, b_{j}>0, \gamma, \gamma_{j} \in[0,1), j=1,2, \ldots, m$, such that

$$
|f(t, u)| \leq a+b|u|^{\gamma}, \quad\left|I_{j}(u)\right| \leq a_{j}+b_{j}|u|^{\gamma_{j}}
$$

for $t \in J$.

Theorem 3.2 Assume that (H1) is satisfied, then system (1.1) has at least one solution $u$, and $u$ minimizes the functional (2.2).

Proof According to (H1), we have

$$
\begin{aligned}
\varphi(u) & =\frac{1}{2}\|u\|^{2}-\int_{0}^{T} F(t, u(t)) d t-\sum_{j=1}^{m} \int_{0}^{u\left(t_{j}\right)} I_{j}(t) d t+\frac{\alpha}{2 \beta} u^{2}(T) \\
& \geq \frac{1}{2}\|u\|^{2}-T\left(a\|u\|_{\infty}+\frac{b}{\gamma+1}\|u\|_{\infty}^{\gamma+1}\right)-\sum_{j=1}^{m}\left(a_{j}\|u\|_{\infty}+\frac{b_{j}}{\gamma_{j}+1}\|u\|_{\infty}^{\gamma_{j}+1}\right)
\end{aligned}
$$




$$
\begin{aligned}
\geq & \frac{1}{2}\|u\|^{2}-T\left(a T^{\frac{1}{2}}\|u\|+\frac{b}{\gamma+1} T^{\frac{\gamma+1}{2}}\|u\|^{\gamma+1}\right) \\
& -\sum_{j=1}^{m}\left(a_{j} T^{\frac{1}{2}}\|u\|+\frac{b_{j}}{\gamma_{j}+1} T^{\frac{\gamma_{j}+1}{2}}\|u\|^{\gamma_{j}+1}\right)
\end{aligned}
$$

for all $u \in H$. This implies that $\varphi$ is coercive.

Let $\left\{u_{n}\right\}$ be a weakly convergent sequence to $u$ in $H$, then $\left\{u_{n}\right\}$ converges uniformly to $u$ in $C[0, T]$.

Set

$$
\begin{aligned}
& \varphi_{1}(u)=-\int_{0}^{T} F(t, u(t)) d t-\sum_{j=1}^{m} \int_{0}^{u\left(t_{j}\right)} I_{j}(t) d t+\frac{\alpha}{2 \beta} u^{2}(T), \\
& \varphi_{2}(u)=\frac{1}{2} \int_{0}^{T}\left[\left(u^{\prime}(t)\right)^{2}+g(t) u^{2}(t)\right] d t,
\end{aligned}
$$

then $\varphi(u)=\varphi_{1}(u)+\varphi_{2}(u)$. So $\varphi_{1}$ is weakly sequentially continuous. Clearly, $\varphi_{2}$ is continuous and convex, which implies that $\varphi_{2}$ is weakly sequentially lower semi-continuous. Therefore, $\varphi$ is weakly sequentially lower semi-continuous on $H$.

By Lemma 2.4, the functional $\varphi$ has a minimum which is a critical point of $\varphi$. Hence, system (1.1) has at least one solution.

Example 3.2 Consider the following boundary value problem:

$$
\left\{\begin{array}{l}
-u^{\prime \prime}(t)+u(t)=1+u(t)^{\frac{1}{3}}, \quad t \neq t_{1}, t \in J=[0,1] \\
-\Delta u^{\prime}\left(t_{1}\right)=1+u(t)^{\frac{1}{5}}, \quad t_{1}=\frac{1}{2} \\
u(0)=0, \quad u(1)+u^{\prime}(1)=0 .
\end{array}\right.
$$

Here $g(t)=1, f(t, u)=1+u(t)^{\frac{1}{3}}, I_{j}(u)=1+u(t)^{\frac{1}{5}}, T=1, j=1, \alpha=1, \beta=1$. Clearly, (H1) is satisfied. Applying Theorem 3.2, problem (3.2) has at least one solution.

\subsection{Existence of at least two distinct solutions}

In this section, we derive some sufficient conditions under which the functional $\varphi$ admits at least two distinct critical points; consequently, (1.1) admits at least two distinct solutions. We first introduce some assumptions.

(H2) $\lim _{u \rightarrow 0} \frac{f(t, u)}{u}=0$ uniformly for $t \in J, \lim _{u \rightarrow 0} \frac{I_{j}(u)}{u}=0$.

(H3) There exist constants $\mu>2$ and $r \geq 0$ such that for every $t \in J$ and $u \in R$ with $|u| \geq r$,

$$
0<\mu F(t, u) \leq u f(t, u), \quad 0<\mu \int_{0}^{u} I_{j}(s) d s \leq u I_{j}(u), \quad j=1,2, \ldots, m
$$

where $F(t, u)=\int_{0}^{u} f(t, s) d s$.

Theorem 3.3 Assume that (H2) and (H3) are satisfied. Then system (1.1) has at least two solutions. 
Proof The proof will be given in three steps.

Step 1. The functional $\varphi$ satisfies the PS condition.

Let $\left\{u_{n}\right\} \subset H$ such that $\left\{\varphi\left(u_{n}\right)\right\}$ is a bounded sequence and $\lim _{n \rightarrow \infty} \varphi^{\prime}\left(u_{n}\right)=0$. We compute

$$
\begin{aligned}
\varphi\left(u_{n}\right)-\frac{1}{\mu} \varphi^{\prime}\left(u_{n}\right)\left(u_{n}\right)= & \left(\frac{1}{2}-\frac{1}{\mu}\right)\left\|u_{n}\right\|^{2}+\left(\frac{1}{2}-\frac{1}{\mu}\right) \frac{\alpha}{\beta} u_{n}^{2}(T) \\
& -\sum_{j=1}^{m} \int_{0}^{u\left(t_{j}\right)} I_{j}(s) d s+\frac{1}{\mu} \sum_{j=1}^{m} I_{j}\left(u_{n}\left(t_{j}\right)\right) u_{n}\left(t_{j}\right) \\
& -\int_{0}^{T} F\left(t, u_{n}(t)\right) d t+\frac{1}{\mu} \int_{0}^{T} f\left(t, u_{n}(t)\right) u_{n}(t) d t \\
\geq & \left(\frac{1}{2}-\frac{1}{\mu}\right)\left\|u_{n}\right\|^{2}+\sum_{j=1}^{m}\left(\frac{1}{\mu} I_{j}\left(u_{n}\left(t_{j}\right)\right) u_{n}\left(t_{j}\right)-\int_{0}^{u\left(t_{j}\right)} I_{j}(s) d s\right) \\
& +\int_{0}^{T}\left(\frac{1}{\mu} f\left(t, u_{n}(t)\right) u_{n}(t)-F\left(t, u_{n}(t)\right)\right) d t .
\end{aligned}
$$

By (H3), one has

$$
\begin{aligned}
\varphi\left(u_{n}\right) & -\frac{1}{\mu} \varphi^{\prime}\left(u_{n}\right)\left(u_{n}\right) \\
\geq & \left(\frac{1}{2}-\frac{1}{\mu}\right)\left\|u_{n}\right\|^{2}-\sum_{j=1}^{m} \max _{u_{n}(t) \in[-r, r]}\left|\frac{1}{\mu} I_{j}\left(u_{n}\left(t_{j}\right)\right) u_{n}\left(t_{j}\right)-\int_{0}^{u\left(t_{j}\right)} I_{j}(s) d s\right| \\
& -\int_{0}^{T} \max _{t \in J, u_{n}(t) \in[-r, r]}\left|\frac{1}{\mu} f\left(t, u_{n}(t)\right) u_{n}(t)-F\left(t, u_{n}(t)\right)\right| d t .
\end{aligned}
$$

Hence, $\left\{u_{n}\right\}$ is bounded in $H$.

From the reflexivity of $H$, we may extract a weakly convergent subsequence; for simplicity, we also note again by $\left\{u_{n}\right\}, u_{n} \rightarrow u$ in $H$. Next we prove that $\left\{u_{n}\right\}$ strongly converges to $u$ in $H$. By $(2.3)$ we have

$$
\begin{aligned}
& \left(\varphi^{\prime}\left(u_{n}\right)-\varphi^{\prime}(u)\right)\left(u_{n}-u\right) \\
& =\left\|u_{n}-u\right\|^{2}+\frac{\alpha}{\beta}\left(u_{n}(T)-u(T)\right)^{2} \\
& \quad-\sum_{j=1}^{m}\left(I_{j}\left(u_{n}\left(t_{j}\right)\right)-I_{j}\left(u\left(t_{j}\right)\right)\right)\left(u_{n}\left(t_{j}\right)-u\left(t_{j}\right)\right) \\
& \quad-\int_{0}^{T}\left(f\left(t, u_{n}(t)\right)-f(t, u(t))\right)\left(u_{n}(t)-u(t)\right) d t .
\end{aligned}
$$

$u_{n} \rightarrow u$ in $H$ implies that $\left\{u_{n}\right\}$ uniformly converges to $u$ in $C[0, T]$. So

$$
\begin{aligned}
& \sum_{j=1}^{m}\left(I_{j}\left(u_{n}\left(t_{j}\right)\right)-I_{j}\left(u\left(t_{j}\right)\right)\right)\left(u_{n}\left(t_{j}\right)-u\left(t_{j}\right)\right) \rightarrow 0, \\
& \int_{0}^{T}\left(f\left(t, u_{n}(t)\right)-f(t, u(t))\right)\left(u_{n}(t)-u(t)\right) d t \rightarrow 0, \\
& u_{n}(T)-u(T) \rightarrow 0 \quad \text { as } n \rightarrow \infty
\end{aligned}
$$


By $\varphi^{\prime}\left(u_{n}\right) \rightarrow 0$ and $u_{n} \rightarrow u$ as $n \rightarrow+\infty$, we have

$$
\left(\varphi^{\prime}\left(u_{n}\right)-\varphi^{\prime}(u)\right)\left(u_{n}-u\right) \rightarrow 0 \quad \text { as } n \rightarrow \infty
$$

So (3.5), (3.6) and (3.7) yield $\left\|u_{n}-u\right\| \rightarrow 0$ in $H$, i.e., $\left\{u_{n}\right\}$ strongly converges to $u$ in $H$. Therefore, the functional $\varphi$ satisfies the PS condition.

Step 2. We show that there exists $\rho>0$ such that the functional $\varphi$ has a local minimum $u_{0} \in B_{\rho}=\{u \in H:\|u\|<\rho\}$.

Firstly, we claim that $\overline{B_{\rho}}$ is bounded and weakly sequentially closed.

In fact, let $\left\{u_{n}\right\} \subseteq \overline{B_{\rho}}$ and $\left\{u_{n}\right\} \rightarrow u$ as $n \rightarrow \infty$. By the Mazur theorem [22], there exists a sequence of convex combinations

$$
v_{n}=\sum_{j=1}^{n} \alpha_{n_{j}} u_{j}, \quad \sum_{j=1}^{n} \alpha_{n_{j}}=1, \quad \alpha_{n_{j}} \geq 0, j \in N
$$

such that $v_{n} \rightarrow u$ in $H .\left\{v_{n}\right\} \subset \overline{B_{\rho}}$ and $u \in \overline{B_{\rho}}$, since $\overline{B_{\rho}}$ is a closed convex set.

Secondly, we claim that the functional $\varphi$ is weakly sequentially lower semi-continuous on $\overline{B_{\rho}}$.

Let

$$
\begin{aligned}
& \varphi_{1}(u)=-\int_{0}^{T} F(t, u(t)) d t-\sum_{j=1}^{m} \int_{0}^{u\left(t_{j}\right)} I_{j}(t) d t+\frac{\alpha}{2 \beta} u^{2}(T), \\
& \varphi_{2}(u)=\frac{1}{2} \int_{0}^{T}\left[\left(u^{\prime}(t)\right)^{2}+g(t) u^{2}(t)\right] d t,
\end{aligned}
$$

then $\varphi(u)=\varphi_{1}(u)+\varphi_{2}(u)$. By $\left\{u_{n}\right\} \rightarrow u$ on $H$, we see that $\left\{u_{n}\right\}$ uniformly converges to $u$ in $C[0, T]$. So $\varphi_{1}$ is weakly sequentially continuous. Clearly, $\varphi_{2}$ is continuous and convex, which implies that $\varphi_{2}$ is weakly sequentially lower semi-continuous. Therefore, $\varphi$ is weakly sequentially lower semi-continuous on $\overline{B_{\rho}}$.

Thirdly, we claim that $\varphi$ has a minimum $u_{0} \in \overline{B_{\rho}}$.

In fact, $H$ is a reflexive Banach space, $\overline{B_{\rho}}$ is bounded and weakly sequentially closed and $\varphi$ is weakly sequentially lower semi-continuous on $\overline{B_{\rho}}$. So, by Lemma 2.4 , there exists $u_{0} \in \overline{B_{\rho}}$ such that $\varphi\left(u_{0}\right)=\min \left\{\varphi(u): u \in \overline{B_{\rho}}\right\}$.

Finally, we claim that $\varphi\left(u_{0}\right)<\inf _{u \in \partial B_{\rho}} \varphi(u)$.

By (H2), let $\varepsilon=\frac{1}{3 m T^{2}}>0$, there exists $\delta>0$ such that $|u|<\delta$ implies

$$
|F(t, u)| \leq \frac{\varepsilon}{2}|u|^{2}, \quad \int_{0}^{u} I_{j}(t) d t \leq \frac{\varepsilon}{2}|u|^{2} \quad \text { for } t \in J
$$

Consequently, by Lemma 2.9 , for $\|u\| \leq \frac{\delta}{\sqrt{T}}$, we have

$$
\begin{aligned}
\varphi(u) & =\frac{1}{2}\|u\|^{2}-\int_{0}^{T} F(t, u(t)) d t-\sum_{j=1}^{m} \int_{0}^{u\left(t_{j}\right)} I_{j}(t) d t+\frac{\alpha}{2 \beta} u^{2}(T) \\
& \geq \frac{1}{2}\|u\|^{2}-\int_{0}^{T} \frac{\varepsilon}{2}|u(t)|^{2} d t-\sum_{j=1}^{m} \frac{\varepsilon}{2}\left|u\left(t_{j}\right)\right|^{2}+\frac{\alpha}{2 \beta} u^{2}(T)
\end{aligned}
$$




$$
\begin{aligned}
& \geq \frac{1}{2}\|u\|^{2}-\frac{\varepsilon T^{2}}{2}\|u\|^{2}-\frac{\varepsilon m T^{2}}{2}\|u\|^{2} \\
& \geq \frac{1}{2}\|u\|^{2}-\frac{1}{6}\|u\|^{2}-\frac{1}{6}\|u\|^{2} \\
& \geq \frac{1}{6}\|u\|^{2}
\end{aligned}
$$

Choose $C=\frac{\delta^{2}}{6 T}, \rho=\frac{\delta}{\sqrt{T}}$, then $\varphi(u) \geq C>0$ for any $u \in \partial B_{\rho}$. Besides, $\varphi\left(u_{0}\right) \leq \varphi(0)=0<$ $C \leq \varphi(u)$ for any $u \in \partial B_{\rho}$. So $\varphi\left(u_{0}\right)<\inf _{u \in \partial B_{\rho}} \varphi(u)$. Hence, $\varphi$ has a local minimum $u_{0} \in$ $B_{\rho}=\{u \in H:\|u\|<\rho\}$.

Step 3. We prove that there exists $u_{1}$ with $\left\|u_{1}\right\|>\rho$ such that $\varphi\left(u_{1}\right)<\inf _{u \in \partial B_{\rho}} \varphi(u)$.

Condition (H3) implies that there exist $b_{1}, b_{2}, c_{j}, d_{j}>0, j=1,2, \ldots, m$, such that

$$
F(t, u) \geq b_{1}|u|^{\mu}-b_{2}, \quad \int_{0}^{u} I_{j}(t) d t \geq c_{j}|u|^{\mu}-d_{j}
$$

for $t \in J, u \in R$ (see [16]). Then we have

$$
\begin{aligned}
\varphi(u) & =\frac{1}{2}\|u\|^{2}-\int_{0}^{T} F(t, u(t)) d t-\sum_{j=1}^{m} \int_{0}^{u\left(t_{j}\right)} I_{j}(t) d t+\frac{\alpha}{2 \beta} u^{2}(T) \\
& \leq \frac{1}{2}\|u\|^{2}-\left(b_{1} T^{\frac{\mu}{2}}\|u\|^{\mu}-b_{2}\right) T-\sum_{j=1}^{m}\left(c_{j} T^{\frac{\mu}{2}}\|T\|^{\mu}-d_{j}\right)+\frac{\alpha}{2 \beta} u^{2}(T) .
\end{aligned}
$$

Since $\mu>2$, (3.10) implies $\lim _{\|u\| \rightarrow \infty} \varphi(u)=-\infty$. Therefore, we can choose $u_{1}$ with $\left\|u_{1}\right\|>\rho$ sufficiently large such that $\varphi\left(u_{1}\right)<\inf _{u \in \partial B_{\rho}} \varphi(u)$.

Let

$$
c=\inf _{h \in \Gamma} \max _{t \in[0,1]} \varphi(h(t))
$$

where

$$
\Gamma=\left\{h \in C([0,1], H): h(0)=u_{0}, h(1)=u_{1}\right\} .
$$

By Lemma 2.6, $c$ is a critical value of $\varphi$, that is, there exists a critical point $u^{*}$. Therefore, $u_{0}, u^{*}$ are two critical points of $\varphi$, and they are solutions of (1.1).

Example 3.3 Consider the following boundary value problem:

$$
\left\{\begin{array}{l}
-u^{\prime \prime}(t)+u(t)=\frac{1}{15}(u(t))^{\frac{8}{3}} \sin t, \quad t \neq t_{j}, t \in J=[0,1] \\
-\Delta u^{\prime}\left(t_{j}\right)=\frac{1}{2} u^{3}\left(t_{j}\right), \quad j=1, \\
u(0)=0, \quad u(1)+u^{\prime}(1)=0 .
\end{array}\right.
$$

Here $g(t)=1, f(t, u)=\frac{1}{15}(u(t))^{\frac{8}{3}} \sin t, I_{j}(u)=\frac{1}{2} u^{3}, T=1, j=1, \alpha=1, \beta=1$. Let $\mu=3, r=1$. Clearly, (H2) and (H3) are satisfied. By Theorem 3.3, problem (3.11) has at least two solutions. 


\subsection{Existence of infinitely many solutions}

In this section, we derive some conditions under which system (1.1) admits infinitely many distinct solutions. To this end, we need the following assumption.

(H4) $f(t, u)$ and $I_{j}, j=1,2, \ldots, m$, are odd about $u$.

Theorem 3.4 Assume that (H2), (H3) and (H4) are satisfied. Then system (1.1) has infinitely many solutions.

Proof We apply Lemma 2.7 to finish the proof. Clearly, $\varphi \in C^{1}(H, R)$ is even since $f(t, u)$ and $I_{j}(u)$ are odd about $u$, and $\varphi(0)=0$. The arguments of Theorem 3.3 show that the functional $\varphi$ satisfies the PS condition. In the same way as in Theorem 3.3, we can easily verify that conditions (i) and (ii) of Lemma 2.7 are satisfied. According to Lemma 2.7, $\varphi$ possesses infinitely many critical points, i.e., system (1.1) has infinitely many solutions.

Example 3.4 Consider the following boundary value problem:

$$
\left\{\begin{array}{l}
-u^{\prime \prime}(t)+u(t)=t u^{3}(t), \quad t \neq t_{j}, t \in J=[0,1] \\
-\Delta u^{\prime}\left(t_{j}\right)=\frac{1}{2} u^{3}\left(t_{j}\right), \quad j=1 \\
u(0)=0, \quad u(1)+u^{\prime}(1)=0
\end{array}\right.
$$

Here $g(t)=1, f(t, u)=t u^{3}, I_{j}(u)=\frac{1}{2} u^{3}, T=1, j=1, \alpha=1, \beta=1$. Obviously, $f(t, u), I_{j}(u)$ are odd about $u$. Let $\mu=3, r=1$. Clearly, (H2) and (H3) are satisfied. Applying Theorem 3.4, problem (3.12) has infinitely many solutions.

\section{Competing interests}

The authors declare that they have no competing interests.

Authors' contributions

ZL and JX conceived of the study and drafted the manuscript. GC participated in the discussion. All authors read and approved the final manuscript.

\section{Author details}

${ }^{1}$ Department of Mathematics, Hunan Normal University, Changsha, Hunan 410081, P.R. China. ${ }^{2}$ College of Mathematics and Statistics, Jishou University, Jishou, Hunan 416000, P.R. China.

\section{Acknowledgements}

The authors are very grateful to the referees for their very helpful comments and suggestions, which greatly improved the presentation of this paper. This work is supported by the Scientific Research Fund of Hunan Provincial Education Department (No: 13K029).

\section{Received: 10 November 2013 Accepted: 4 April 2014 Published: 06 May 2014}

\section{References}

1. Bainov, DD, Simeonov, PS: Impulsive Differential Equations: Periodic Solutions and Applications. Longman Scientific and Technical, Harlow (1993)

2. Lakshnikantham, V, Bainov, DD, Simeonov, PS: Theory of Impulsive Differential Equations. World Scientific, Singapore (1989)

3. Ding, W, Mi, JR, Han, MA: Periodic boundary value problems for the first order impulsive functional differential equations. Appl. Math. Comput. 165, 433-446 (2005)

4. Hristova, SG, Kulev, GK: Quasilinearization of a boundary value problem for impulsive differential equations. J. Comput. Appl. Math. 132, 399-407 (2001)

5. Luo, ZG, Nieto, JJ: New results for the periodic boundary value problems for impulsive integro-differential equations. Nonlinear Anal. 70, 2248-2260 (2009)

6. Li, JL: Periodic boundary value problems for second order impulsive integro-differential equations. Appl. Math. Comput. 198, 317-325 (2008) 
7. Ladde, GS, Lakshmikantham, V, Vatsala, AS: Monotone Iterative Techniques for Nonlinear Differential Equations. Pitman, London (1985)

8. Rachunkova, I, Tvrdy, M: Existence results for impulsive second-order periodic problems. Nonlinear Anal. 59, 133-146 (2005)

9. Chen, L, Sun, J: Nonlinear boundary vale problem of first order impulsive functional differential equations. J. Math. Anal. Appl. 318, 726-741 (2006)

10. He, ZM, He, XM: Monotone iterative technique for impulsive integro-differential equations with periodic boundary conditions. Comput. Math. Appl. 48, 73-84 (2004)

11. Nieto, JJ, Regan, DO: Variational approach to impulsive differential equations. Nonlinear Anal., Real World Appl. 10, 680-690 (2009)

12. Qian, DB, Li, XY: Periodic solutions for ordinary differential equations with sublinear impulsive effects. J. Math. Anal. Appl. 303, 288-303 (2005)

13. Rachunkova, l, Tvrdy, M: Non-order lower and upper function in second order impulsive periodic problems. Dyn. Contin. Discrete Impuls. Syst., Ser. A Math. Anal. 12, 397-415 (2005)

14. Zuo, WJ, Jiang, DQ, Regan, DO, Agarwal, RP: Optimal existence conditions for the periodic delay $\Phi$-Laplace equation with upper and lower solutions in the reverse order. Results Math. 44, 375-385 (2003)

15. Tian, Y, Ge, WG: Applications of variational methods to boundary-value problem for impulsive differential equations. Proc. Edinb. Math. Soc. 51, 509-527 (2008)

16. Tian, Y, Wang, J, Ge, WG: Variational methods to mixed boundary value problem for impulsive differential equations. Taiwan. J. Math. 13, 1353-1370 (2009)

17. Xie, JL, Luo, ZG: Solutions to a boundary value problem of a fourth-order impulsive differential equation. Bound Value Probl. 2013, 154 (2013)

18. Zhang, H, Li, ZX: Variational approach to impulsive differential equations with periodic boundary conditions. Nonlinear Anal., Real World Appl. 11, 67-78 (2010)

19. Zhang, ZH, Yuan, R: An application of variational methods to Dirichlet boundary value problem with impulses. Nonlinear Anal., Real World Appl. 11, 155-162 (2010)

20. Mawhin, J, Willem, M: Critical Point Theory and Hamiltonian Systems. Springer, Berlin (1989)

21. Chipot, M: Elements of Nonlinear Analysis. Birkhäuser, Basel (2000)

22. Kaus, D: Nonlinear Functional Analysis. Dover Publications, Dover (2009)

23. Rabinowitz, PH: Minimax Methods in Critical Point Theory with Applications to Differential Equations. CBMS Regional Conference Series in Mathematics, vol. 65. Am. Math. Soc., Providence (1986)

24. Zhou, JW, Li, YK: Existence and multiplicity of solutions for some Dirichlet problems with impulsive effects. Nonlinear Anal. 71, 2856-2865 (2009)

10.1186/1687-1847-2014-118

Cite this article as: Luo et al.: Existence of solutions of a second-order impulsive differential equation. Advances in

Difference Equations 2014, 2014:118

\section{Submit your manuscript to a SpringerOpen ${ }^{\ominus}$ journal and benefit from:}

- Convenient online submission

- Rigorous peer review

- Immediate publication on acceptance

- Open access: articles freely available online

- High visibility within the field

- Retaining the copyright to your article 